ISSN 0001-6002/2009/51/4/206-212 Acta Médica Costarricense, (C2009 Colegio de Médicos y Cirujanos

\title{
Revisión
}

\section{Perfil oxidativo de la mujer menopáusica: Papel de los estrógenos en la prevención y tratamiento de las enfermedades}

\section{(Oxidative Profile of the Menopausal Woman: Estrogens' Rol in the Prevention and Treatment of Diseases)}

\author{
Carlos Escalante-Gómez, Silvia Quesada-Mora, Fernando Zeledón-Sánchez
}

\section{Resumen}

El balance entre la producción de radicales libres de oxigeno y el nivel de enzimas antioxidantes es permanentemente regulado por la célula. Las enzimas antioxidantes son esenciales para las células aeróbicas, puesto que mantienen en niveles aceptables las concentraciones de especies químicas conocidas como radicales libres. Durante el climaterio o etapa perimenopáusica, el cuerpo y metabolismo de una mujer cambia de forma importante, y los mecanismos que regulan el estrés oxidativo no son la excepción. Los estrógenos, per se, son antioxidantes, y esto ha impulsado recientemente estudios sobre los efectos antioxidativos de los estrógenos, como un posible mecanismo preventivo para algunas enfermedades. En los últimos años se le ha brindado gran importancia al papel del estrés oxidativo en la génesis de algunas enfermedades como la ateroesclerosis y la enfermedad cardiovascular, algunos tipos de cánceres y la enfermedad de Alzheimer. Pareciera que los estrógenos sí modifican el equilibro oxidativo / antioxidativo in vivo, sin embargo aun se requieren más estudios para puntualizar el rol de estos como posibles tratamientos preventivos para la menopáusica.

Descriptores: estrés oxidativo, antioxidantes, menopausia, estrógenos

Departamento de Bioquímica, Universidad de Costa Rica,

Programa de Maestría en Ciencias Biomédicas Abreviaturas: CAT, catalasa; $\mathrm{Cu} / \mathrm{Zn}$-SOD, $\quad$ superóxido dismutasa citosólica; EcSOD, superóxido dismutasa extracelular; GSH, glutatión; GSH-PX, glutatión peroxidasa; LDL, lipoproteína de baja densidad; LPO, hidroperóxido lipídico; MDA, maldonaldehido; MnSOD, superóxido dismutasa mitocondrial; ROS, especies reactivas de oxígeno; SOD, superóxido dismutasa; TAS, estatus antioxidativo total; TBARS, ácido tiobarbitúrico; $\mathrm{TRH}$, terapia de reemplazo hormonal

Correspondencia: Carlos Escalante Gómez Apto. 783-2400, San José, Costa Rica Email: dr.escalante@gmail.com

\section{Abstract}

The cell carefully regulates the balance between the production of radical oxygen species and antioxidant enzymes levels. Antioxidant enzymes are critical for aerobic cells to maintain within balance free radical production. During the climacteric transition, the body and its metabolism suffer many changes and the mechanisms that regulate oxidative balance are not the exception. Estrogens, per se, are antioxidants, and this has promoted several studies to investigate the potential role of estrogens in disease prevention. Within the last few years, a great deal of studies have shown the relationship between oxidative stress and diseases such as atherosclerosis and cardiovascular disease, cancer and Alzheimer's disease. It appears that estrogens do modify the oxidative / anti-oxidative equilibrium in vivo, but more investigation is needed to further specify their role in disease prevention during the climacteric phase.

Key Words: oxidative stress, antioxidants, menopause, estrogens

Recibido: 9 de febrero de 2009

Aceptado: 10 de marzo de 2009 
Hoy, los estudios acerca de los radicales libres han mostrado cómo estas pequeñas moléculas participan en todos los procesos bioquímicos del cuerpo humano. El envejecimiento, visto desde el punto de vista fisiológico, es un proceso en donde el equilibrio oxidativo cambia drásticamente según la etapa estudiada, y el climaterio no es la excepción. Dentro de la etapa climatérica se observan cambios en el comportamiento oxidativo de la persona, los cuales podrían estar en relación directa con algunos trastornos de la menopausia. Al tener un concepto más claro acerca de lo que son los procesos oxidativos y antioxidativos del cuerpo, se puede analizar el climaterio como una etapa de desequilibrio oxidativo. No solo se han evidenciado cambios oxidativos en el climaterio, sino que también se ha mostrado la modificación de estos con la terapia de reemplazo hormonal. En esta revisión se abarcan conceptos básicos del proceso oxidativo y se enfocan los cambios oxidativos de la etapa climatérica. Se mencionan los estudios actuales, que han mostrado influencia del estado oxidativo con la terapia de reemplazo hormonal, y cómo estos abren el camino a futuras investigaciones.

\section{Radicales libres y antioxidantes}

Para controlar la producción de especies reactivas de oxígeno (ROS) en la célula, esta cuenta con un complejo de enzimas que funcionan como antioxidantes y son moléculas que degradan o transforman los radicales libres, generando productos no tóxicos. El balance entre la producción de ROS $y$ el nivel de enzimas antioxidantes es permanentemente regulado por la célula.

Las enzimas antioxidantes son esenciales para las células aeróbicas, puesto que mantienen en niveles aceptables las concentraciones de especies químicas conocidas como radicales libres, los cuales se caracterizan por presentar un electrón desapareado y por ser muy reactivos. El hecho de que la célula disponga en abundancia del dispositivo defensivo constituido por las enzimas antioxidantes, evidencia el importante grado de toxicidad que poseen los radicales libres. ${ }^{1}$

Durante el metabolismo aerobio se generan pequeñas cantidades de ROS, aniones superóxido $\left(\mathrm{O}_{2}^{-}\right)$y peróxido de hidrógeno $\left(\mathrm{H}_{2} \mathrm{O}_{2}\right)$, como respuesta a estímulos externos e internos. Estas mínimas concentraciones de ROS pueden ser indispensables en muchos procesos, como el sistema de señales intracelulares (relacionado con otros procesos como la proliferación celular y la apoptosis), la inmunidad y la defensa contra microorganismos. ${ }^{2}$

Sin embargo, una producción elevada de ROS puede dar lugar a estrés oxidativo, causante de graves disfunciones metabólicas y daño a macromoléculas biológicas. ${ }^{1}$ Existe, por lo tanto, una relación entre los niveles de las enzimas antioxidantes y los tres tipos de moléculas mensajeras (factores de crecimiento, prostaglandinas y óxido nítrico) implicadas en la homeostasis celular, es decir, un equilibrio entre el mantenimiento de las condiciones estáticas o constantes en el medio intracelular y el nivel de ROS. ${ }^{3}$

Actualmente, se le otorga la importancia de toxicidad que conllevan los radicales libres durante los procesos biológicos, en los cuales una de las consecuencias del estrés oxidativo es la peroxidación lipídica, cuya prevención es esencial en todos los organismos aerobios, ya que los productos derivados de este proceso pueden interactuar con el ADN y son potencialmente mutágenos. Los epóxidos formados pueden reaccionar de manera espontánea con centros nucleofílicos en la célula, o unirse a los ácidos nucléicos (ADN y ARN). Esta reacción puede dar lugar a citotoxicidad, alergia, mutagénesis o carcinogénesis, dependiendo de las propiedades del epóxido en cuestión. ${ }^{3,4}$

En los organismos aerobios existe una gran variedad de sistemas de defensa antioxidante, tanto enzimáticos como no enzimáticos, que se coordinan en forma cooperativa y protegen al organismo de los riesgos que conlleva el estrés oxidativo. Entre ellos destacan las actividades enzimáticas superóxido dismutasa (SOD), glutatión peroxidasa (GSHPX), catalasa (CAT), y el glutatión (GSH), además del ácido ascórbico (vitamina C), alfatocoferol (vitamina E), betacaroteno, vitamina A, flavonoides y compuestos fenólicos. ${ }^{1-4}$

Por otro lado, también hay una relación entre los niveles de ROS celulares y el incremento o descenso de las actividades de las enzimas antioxidantes. El descontrol de todas estas especies reactivas de oxígeno puede, por lo tanto, afectar diferentes procesos esenciales del organismo, y constituirse en una de las piedras angulares en la génesis de distintas patologías.

\section{Estrés oxidativo y enfermedades}

En los últimos años se le ha dado gran importancia al papel del estrés oxidativo en la génesis de algunas enfermedades, como la ateroesclerosis y la enfermedad cardiovascular, ${ }^{5-9}$ varios tipos de cánceres, ${ }^{9-12}$ la enfermedad deAlzheimer, ${ }^{13,14}$ las cataratas ${ }^{15}$ y ciertas inmunodeficiencias. ${ }^{16}$ Sin lugar a duda, la relación entre alteraciones en el equilibro oxidativo y la patogénesis de las enfermedades cardiovasculares, parece ser la más importante desde el punto de vista epidemiológico. Según las estadísticas del Ministerio de Salud de Costa Rica, las enfermedades cardiovasculares han cobrado más vidas en los últimos diez años, que todas las enfermedades malignas sumadas. ${ }^{17} \mathrm{La}$ cantidad de defunciones por causa circulatoria en América Latina, representa casi el $50 \%$ de las muertes por enfermedades no transmisibles, ${ }^{18}$ y es la principal causa de muerte en los Estados Unidos, en Europa y en Japón. ${ }^{8}$ Solo en Estados Unidos, se estima que en 2006 se han gastado 406 mil millones de dólares para atender las complicaciones de las enfermedades cardiovasculares. 
Los mecanismos por los cuales el estrés oxidativo participa en la génesis de la aterosclerosis están bien definidos. ${ }^{19}$ Las altas concentraciones de LDL plasmático favorecen que estas moléculas penetren los vasos en sitios de previa lesión, de manera que se alojan en la íntima de los vasos. Aquí, estas moléculas de LDL son oxidadas por macrófagos locales y luego son ingeridas por estos mismos macrófagos para formar células espumosas, base de las estrías lipídicas de la aterosclerosis. Estas moléculas de LDL oxidadas también cumplen otras funciones patogénicas: 1) aumentan el reclutamiento de monocitos circulantes hacia la íntima de los vasos, incrementando la lesión; 2) inhiben el retorno de macrófagos hacia el lumen vascular; y 3) las moléculas per se, de LDL oxidadas pueden causar lesión celular local por medio de radicales libres. ${ }^{19,20}$ Aunque no se ha podido confirmar si estos mecanismos son un factor causal único, o más bien uno de muchos factores que favorecen la formación de ateroesclerosis, sí se ha demostrado que las placas ateromatosas humanas contienen productos de peroxidación lipídica no presentes en otros vasos. También se ha comprobado que los niveles de LDL oxidados son mayores en pacientes con enfermedad ateroesclerótica, y que estos mismos pacientes presentan anticuerpos contra LDL oxidados. ${ }^{21}$

El estudio WHO/MONICA, ${ }^{22}$ realizado con muestras de 16 países, evidenció que los niveles de $\alpha$-tocoferol en plasma presentaban una correlación inversa con el índice de muertes por enfermedad cardiovascular. El estudio Nurses Health Study, ${ }^{23}$ que incluyó a 87245 mujeres sanas, entre la edad de 34-59, mostró que el grupo con mayor consumo de vitamina E, presentó un $23-50 \%$ menos incidencia de enfermedad cardiovascular, en un periodo de seguimiento de 8 años. La investigación $\mathrm{CHAOS}^{24}$ estudió el efecto de suplementación de vitamina E, en un grupo de 2000 pacientes con evidencia de enfermedad ateroesclerótica, y mostró una disminución en la incidencia de infartos al miocardio no fatales, de un $47 \%$. Sin embargo, otros estudios como el HOPE, ${ }^{25}$ que incluyó a más de 9000 pacientes en alto riesgo para enfermedad cardiovascular, no logró mostrar beneficio alguno con la administración de vitamina $\mathrm{E}$ como antioxidante. El último gran estudio publicado con respecto a este tema es el Women's Health Study, ${ }^{26}$ que valoró la utilidad de suplementación de vitamina $\mathrm{E}$ en la prevención de eventos cardiovasculares en 40000 mujeres sanas; este estudio no logró mostrar beneficio alguno de la vitamina $\mathrm{E}$ en la prevención de enfermedad cardiovascular. En una revisión sistemática del tema, se evidenció que en estudios observacionales, las personas con un alto consumo dietético de vitaminas antioxidantes presentaban menos incidencia de enfermedad cardiovascular, sin embargo, esta misma revisión rescata que en los ensayos clínicos con vitaminas antioxidantes no se observó esta disminución en la incidencia de enfermedades cardiovasculares. ${ }^{8}$ Según la fisiopatología de la enfermedad, pareciera que los antioxidantes participarían más en la prevención y no en el tratamiento de la enfermedad, pero aun hay mucho por definir.
Recientemente, se ha mostrado que los estrógenos modulan los procesos oxidativos y antioxidativos, logrando una disminución en la producción de radicales libres, ${ }^{27}$ un aumento en la expresión de enzimas antioxidativas ${ }^{27,28} \mathrm{y}$, a la vez, participando como una molécula antioxidante en sí. ${ }^{29}$ Es muy llamativo que luego de la menopausia, cuando bajan los niveles de estrógenos en la mujer, la incidencia de muchas enfermedades asociadas a posibles causas oxidativas, aumenta notablemente. ${ }^{18,30}$ Es de suponer que al disminuir los niveles estrogénicos en la mujer, el equilibro oxidativo se inclinaría hacia el estrés oxidativo, exponiendo a la mujer a una mayor cantidad de noxas propiciadoras de enfermedades. Todavía no se ha realizado un estudio que abarque simultáneamente mediciones de producción de estrés oxidativo como niveles de enzimas antioxidativas en la mujer posmenopáusica para establecer el estado de tal población. Más importante, se ha sugerido que el reemplazo hormonal con estrógenos en modelos animales posmenopáusicas (monas), podría restablecer el equilibrio oxidativo / antioxidativo, y así prevenir o retrasar la aparición de algunas enfermedades como las cardiovasculares. ${ }^{31}$

\section{Equilibrio oxidativo y la menopausia}

Durante el climaterio o etapa perimenopáusica, el cuerpo y metabolismo de una mujer cambia de forma importante, $y$ los mecanismos que regulan el estrés oxidativo no son la excepción. En los últimos años se han realizado estudios que ligan esta disminución en los niveles de estrógenos, con cambios en el comportamiento oxidativo / antioxidativo de la mujer, sin embargo, el panorama aun no está claro.

En estudios con animales se han evidenciado estos cambios; Strehlow y cols. ${ }^{28}$ mostraron una disminución de la superóxido dismutasa extracelular (ecSOD) y la superóxido dismutasa mitocondrial (MnSOD), y un aumento de producción de radicales libres en ratas, asociado a niveles decrecientes de estrógenos, luego de ser ooforectomizadas. Mientras que Wassmann y cols..$^{27}$ evidenciaron un incremento en la actividad de NADPH oxidasa (el cual cataliza la transferencia de un electrón desde el NADPH hacia el $\mathrm{O}_{2}$ en la cadena respiratoria) y en la producción de la superóxido dismutasa en ratas, luego de ser ooforectomizadas.

Recientemente, Signorelli y cols, ${ }^{32}$ lograron demostrar que el nivel de estrés oxidativo, representado por los niveles plasmáticos de 4-hidroxynenal (4-HNE) -el cual es un producto de desecho de la oxidación de los lípidos-, el maldonaldehído (MDA) y la capacidad oxidativa de LDL del plasma, estaba aumentado significativamente en la mujer posmenopáusica comparada con la "fértil". Algo digno de destacar del estudio, es que se efectuó un análisis de características de las poblaciones, y se descartó la edad como factor causal del incremento de estrés oxidativo. Utilizando los niveles de hidroperóxido lipídico (LPO), como indicador de producción de radicales libres y de daño oxidativo de los lípidos de la membrana, Bednarek-Tupikowska y cols. ${ }^{33}$ demostraron que había un mayor nivel de lesión o estrés 
oxidativo en la postmenopáusica comparada con la premenopáusica. Además, en este mismo estudio, se evidenció una relación inversa estadísticamente significativa entre los niveles de estrógeno y los de estrés oxidativo, es decir, a menor nivel de estrógeno, mayor evidencia de lesión oxidativa. Pero, otros estudios ${ }^{34,35}$ no lograron mostrar diferencia significativa entre los niveles de estrés oxidativo de mujeres pre y postmenopáusicas, utilizando como indicador de estrés los niveles de sustancias reactivas al ácido tiobarbiturico (TBARS) en plasma, y tampoco evidenciaron una relación inversa entre los niveles estrogénicos y los indicadores de estrés oxidativo.

Signorelli $^{32}$ evidenció menor actividad del antioxidante glutatión peroxidasa (GSH-PX) en el grupo de mujeres postmenopáusicas, comparado con el grupo de premenopáusicas. Unfer, ${ }^{34}$ en un estudio más detallado, evidenció que los niveles totales de SOD eran significativamente menores en postmenopáusicas que en premenopáusicas. Sin embargo, no encontró diferencias significativas en los niveles de GSH-PX, ni de catalasa (CAT) entre los grupos de pre y postmenopáusicas. Otros autores $^{33,36}$ midieron el estatus antioxidativo total, lo que es una medición general de la capacidad antioxidativa del cuerpo, (TAS) de un grupo de 55 mujeres, y evidenciaron que el grupo postmenopáusico tenía niveles de TAS significativamente menores que el grupo premenopáusico, y a la vez lograron correlacionar la disminución de estrógenos y la disminución de TAS.

Según los estudios anteriores, pareciera que la menopausia se asocia con niveles aumentados de estrés oxidativo y niveles disminuidos de antioxidantes enzimáticos. Aun no se sabe claramente cuáles son los mecanismos fisiológicos de estos cambios, pero algunos autores los han asociado con el incremento de enfermedades en el periodo postmenopáusico.

\section{Efecto antioxidante de los estrógenos}

En los últimos diez años, la terapia de reemplazo hormonal (TRH), como tratamiento de los síntomas climatéricos, ha tenido un gran auge. Además de sus efectos sobre los síntomas climatéricos, la terapia de reemplazo con estrógenos exógenos se ha mostrado beneficiosa en algunas patologías como la osteoporosis. ${ }^{37,38}$ Los posibles efectos cardioprotectores de la terapia de reemplazo estrogénica todavía no están bien definidos, ${ }^{40}$ inclusive los últimos estudios no han logrado evidenciar este efecto y, más bien, han sugerido que hasta podrían tener un efecto perjudicial sobre el aparato cardiovascular. ${ }^{34}$ Esta controversia de los efectos cardioprotectores de los estrógenos ha impulsado recientemente estudios sobre los efectos antioxidativos de los estrógenos como un posible mecanismo preventivo de enfermedades cardiovasculares.

Los estrógenos per se, son antioxidantes, ${ }^{29,}{ }^{41-43}$ ya que poseen un anillo fenol, el cual puede actuar como un barredor de radicales libres $\mathrm{y}$, a la vez, le permite donar un átomo $\mathrm{H}+{ }^{35,42}$ Esta propiedad le posibilita al estrógeno intervenir en diferentes etapas de la oxidación lipídica. Estudios in vitro han evidenciado la capacidad antioxidativa de los estrógenos, al disminuir la oxidación de $\mathrm{LDL}_{\text {y }}$ el $\mathrm{CuSO}_{4}$, e inclusive se ha mostrado una disminución de lesiones inducidas por radicales libres en cadenas de ADN. ${ }^{36}$ Se ha evidenciado que los diferentes estrógenos y sus metabolitos poseen distintas capacidades antioxidativas. ${ }^{44,45}$ En un estudio comparativo in vitro entre algunos tipos de estrógenos, se logró clasificar la capacidad antioxidativa de mayor a menor, así: estradiol $>$ estrona $>$ equilin $>$ estriol. ${ }^{45}$ Mientras que Subbiah $^{42}$ clasifica los estrógenos equinos como los de mayor capacidad antioxidativa, el estradiol y la estrona, junto a sus metabolitos catecol, han mostrado capacidad antioxidativa en concentraciones micromolares, en tanto los metabolitos metoxi mostraron la misma capacidad antioxidativa a concentraciones de picomolares. ${ }^{44}$ Los metabolitos estrogénicos, catecolestrógenos y metoxiestrógenos, además de funcionar como barredores de radicales libres, también lograron reducir y mantener reducidas las moléculas de $\mathrm{Fe} \mathrm{y} \mathrm{Cu}$, lo cual previene que estas moléculas actúen como oxidantes. ${ }^{44}$ Otro estudio ha evidenciado que el estradiol es igual de efectivo que la vitamina $\mathrm{E}$, en prevenir la oxidación de $\mathrm{LDL},{ }^{46}$ e igual o más efectivo que las enzimas SOD y CAT.

Los estrógenos no solo participan como antioxidantes per se, sino que también pueden modificar los niveles $\mathrm{y}$ capacidades de los mecanismos oxidativos y antioxidativos del cuerpo. Strehlow, ${ }^{28}$ logró mostrar que el estradiol disminuía la producción de radicales libres inducido por la angiotensina II en cultivos celulares de músculo liso. Wassmann ${ }^{27}$ concluyó que el estradiol aumenta la transcripción, expresión y actividad de MnSOD y de ecSOD, sin afectar los niveles o actividad de la $\mathrm{Cu}$-ZnSOD, GSHPX ni catalasa. Ayres y cols..$^{41}$ mostraron que los estrógenos podían disminuir la producción de superóxido en células endoteliales de corazones bovinos.

Otro factor que se tiene que tomar en cuenta es que la terapiadereemplazohormonal(TRH)incluyelaadministración de progestágenos cuando la mujer aun tiene útero. Esto propicia la confusión, dado que hay estudios que sugieren que la progesterona tiene efecto antioxidativo, mientras otros afirman que no lo tiene, y otros que, inclusive, le atribuyen efecto prooxidativo. Wassmann y cols. ${ }^{27}$ estudiaron los efectos de la progesterona en cultivos celulares de músculo liso vascular de rata, y mostraron que la progesterona disminuía los niveles, actividad, y transcripción de la ecSOD y MnSOD. También se evidenció que la progesterona revertía los efectos beneficiosos de los estrógenos sobre la ecSOD y MnSOD, y aumentaba la producción endógena de especies reactivas de oxígeno en el músculo liso vascular. Arteaga y cols, ${ }^{47}$ refirieron que los diferentes tipos de progestágenos, por sí solos, no modificaban la oxidación de LDL in vitro. También se ha estudiado el efecto antioxidativo de los estrógenos junto a los 
progestágenos, mostrando que el efecto antioxidativo observado por los estrógenos no fue modificado por el norgestrel ni la noretidiona, pero sí por la progesterona y acetato de medroxiprogesterona, los cuales en dosis altas, más bien potenciaron la capacidad antioxidativa del estrógeno.

Dado el hecho de que en la menopausia hay un cambio en el balance oxidativo/antioxidativo, y que se ha demostrado que los estrógenos pueden actuar como antioxidantes in vitro, algunos autores han estudiado el impacto que la terapia de reemplazo podría tener en el balance oxidativo / antioxidativo de la postmenopáusica.

Ke y Todd ${ }^{48}$ estudiaron el efecto de la TRH (estrógenos + progesterona) sobre la producción de estrés oxidativo en la postmenopáusica. Utilizaron el 8-epi-prostaglandina $\mathrm{F}_{2 \alpha}$ como indicador de oxidación del acido araquidónico de los fosfolípidos de membranas, y valoraron sus niveles después de 6 semanas de TRH. Ellos observaron una disminución de los niveles de 8-epi-prostaglandina $\mathrm{F}_{2 \alpha}$ y concluyeron que la TRH, inclusive en periodos cortos, disminuye el estrés oxidativo en las postmenopáusicas.

McManus y cols, ${ }^{49}$ estudiaron el efecto de diferentes tipos de TRH sobre la capacidad oxidativa del plasma de mujeres postmenopáusicas, y no lograron evidenciar diferencias significativas entre la capacidad de oxidación de LDL del plasma de mujeres que recibían TRH, de las que no lo recibían, llegando a la conclusión de que "los estrógenos no proporcionaban un efecto antioxidativo in vivo".

Inal y cols, ${ }^{50}$ midieron los niveles de SOD, GSH-PX y MDA en un grupo de mujeres que iniciaba TRH solo con estrógenos y un grupo que lo iniciaba con estrógenos y progesterona. Tras seis meses de tratamiento, el estudio no mostró mejoría significativa en la capacidad antioxidativa en ninguno de los dos grupos.

Özden y cols, ${ }^{51}$ midieron los niveles plasmáticos de TBARS y los niveles eritrocitarios de TBARS, GSH-PX y GSH en postmenopáusicas con y sin TRH. El estudio demostró que el grupo de mujeres con TRH presentaban niveles de TBARS plasmáticos y eritrocitarios más bajos que el grupo sin TRH. También se evidenció que el grupo de mujeres con TRH presentaba niveles de GSH-PX y GSH eritrocitarios más altos que el grupo que no lo usaba. Özden comparó las subpoblaciones de TRH y no logró encontrar diferencias significativas entre los grupos de mujeres que recibían solo estrógenos o estrógenos y progesterona.

Unfery cols, ${ }^{34}$ compararon un grupo depostmenopáusicas con y $\sin$ TRH, sin lograr evidenciar diferencias significativas entre niveles plasmáticos de CAT, GSH-PX, ni TBARS; sin embargo, sí refirió niveles mayores de SOD en el grupo que recibía TRH. Inclusive, concluyó que había una tendencia a niveles mayores de SOD en las mujeres que recibían TRH con progesterona, frente a las que solo recibían estrógenos.
Bednarek-Tupikowska ${ }^{33}$ evidenciaron niveles menores de LPO y niveles mayores de TAS, en un grupo de mujeres postmenopáusicas con TRH, en comparación con un grupo que no recibía TRH. También analizaron las subpoblaciones de TRH y no encontraron diferencias entre las mujeres que recibían solo estrógenos y las que recibían estrógenos y progesterona.

Naziroglu y cols, ${ }^{52}$ lograron hacer una comparación más amplia entre postmenopáusicas "sanas" con y sin TRH (estrógenos + progesterona), y postmenopáusicas diabéticas con y sin TRH (estrógenos + progesterona). En el grupo de mujeres postmenopáusicas "sanas", la utilización de TRH por 6 semanas no causó aumento significativo en los niveles plasmáticos de GSH-PX, GSH ni catalasa, sin embargo, sí logró una disminución significativa de MDA. Pero, en el grupo de mujeres postmenopáusicas diabéticas, la utilización de TRH por 6 semanas sí causó un aumento significativo en los niveles plasmáticos de GSH-PX, GSH y catalasa, y también logró una disminución significativa de MDA. También estudiaron el efecto antioxidativo en el nivel eritrocitario. En el grupo de postmenopáusicas "sanas", la utilización de TRH por 6 semanas sí causó aumento significativo en los niveles eritrocitarios de GSH-PX, pero no de GSH ni catalasa, y también logró una disminución significativa de MDA. En el grupo de postmenopáusicas diabéticas, la utilización de TRH por 6 semanas logró un aumento significativo en los niveles eritrocitarios de GSHPX, GSH y catalasa, y como era de esperar, logró una disminución significativa de MDA.

Wen y cols, ${ }^{53}$ siguieron a 18 mujeres que iniciaron TRH (estrógenos + progesterona) y les midieron la capacidad oxidativa de LDL del plasma, a los 3 y 6 meses de tratamiento, y tampoco lograron demostrar diferencia en la capacidad oxidativa de las mujeres, antes y después de TRH durante 6 meses.

Bureau y cols ${ }^{54}$ estudiaron el impacto de la TRH (estrógenos + progesterona) sobre la capacidad antioxidativa en la postmenopáusica. Midieron los niveles de Zn-CuSOD y GSH-PX en eritrocitos de mujeres con TRH y otras que recibían placebo; no se logró mostrar diferencia en la capacidad antioxidativa entre las que recibían TRH y las que no.

Bureau y cols, ${ }^{55}$ en otro estudio, compararon los niveles de TBARS, capacidad de oxidación de LDL y TAS, en postmenopáusicas con o sin TRH(estrógenos + progesterona). El estudio no mostró diferencia en la capacidad de oxidación de LDL entre los grupos; lo sorprendente es que el grupo con TRH mostró niveles de TBARS significativamente mayores, $\mathrm{y}$ niveles de TAS significativamente menores que el grupo de mujeres sin TRH. En un estudio aparte pero relacionado con el tema, Strehlow y cols, ${ }^{28}$ estudiaron los cambios agudos de algunos antioxidantes en pacientes en tratamiento de infertilidad, para analizar el impacto de un aumento súbito en los niveles endógenos de estrógenos. Ellos mostraron que 
en pacientes en quienes se inducía la ovulación, había aumentos súbitos de los niveles de ecSOD y MnSOD, relacionados con el aumento en el nivel de estrógenos.

Lo difícil de analizar el efecto general de los estrógenos con o sin progestágenos sobre el equilibro oxidativo/ antioxidativo in vivo, es que existen diferentes técnicas para medir estos efectos ${ }^{56-65}$ y que la gran mayoría de los autores estudian solo uno o dos componentes del sistema antioxidativo. Esto resulta en estudios que a veces se contradicen entre ellos y que más bien dejan preguntas sobre los componentes no estudiados. Pareciera que los estrógenos sí modifican el equilibro oxidativo / antioxidativo in vivo, al disminuir un poco el estrés oxidativo y a la vez, por mejorar los niveles y actividad de algunos antioxidantes como SOD. Igual que en la población menopáusica sin TRH, no existen estudios que analicen indicadores totales e individuales de estrés oxidativo junto a niveles totales e individuales de antioxidantes, logrando establecer si se presentan cambios asociados a la TRH. También queda por definir si el agregar progestágenos a los estrógenos influye o no sobre el equilibrio oxidativo / antioxidativo final de la mujer. Hay algunos estudios que investigan estas variables, pero no se sabe cuál será el verdadero papel de los estrógenos en la prevención y tratamiento de las enfermedades ligadas al estrés oxidativo.

\section{Referencias}

1. Blokhina O, Virolainen E, Fagerstedt KV. Antioxidants, oxidative damage and oxygen deprivation stress: a review. Ann Bot (Lond) 2003; 91: 179-194.

2. Angstadt C. Estructura de las macromoléculas. En: Bioquímica: libro de texto con aplicaciones clínicas. $4^{\mathrm{a}}$ ed. España: Reverté. 2004; 40 -98 .

3. Murray R. Bioenergética y el metabolismo de carbohidratos y lípidos. En: Bioquímica de Harper. $15^{\text {va }}$ ed. México: El Manual Moderno, 2001; 88-149.

4. Young IS, Woodside JV. Antioxidants in health and disease. J Clin Pathol. 2001; 54: 176-186.

5. Scandalios JG. Oxidative stress: molecular perception and transduction of signals triggering antioxidant gene defensas. Braz J Med Re. 2005; 38: 995-1014.

6. Kyaw M, Yoshizumi M, Tsuchiya K, Izawa Y, Kanematsu Y, Tamaki T. Atheroprotective effects of antioxidants through inhibition of mitogen-activated protein kinases. Acta Pharmacol Sin. 2004; 25: 977-985.

7. Paolisso G, Esposito R, D’Alessio MA, Barbieri M. Primary and secondary prevention of atherosclerosis: is there a role for antioxidants? Diabetes Metab. 1999; 25: 298-306.

8. Willcox, J, Ash S, Catignani G. Antioxidants and prevention of chronic disease. Crit Rev Food Sci Nutr. 2004; 44: 275-295.

9. Tribble DL, Frank E. Dietary antioxidants, cancer and atherosclerotic heart disease. West J Med. 1994; 161:605-612.

10. Ebell MH. Efficacy of antioxidants in GI cancer prevention. Am Fam Physician. 2005; 71: 465-466.
11. Salaganik RL. The benefits and hazards of antioxidants: controlling apoptosis and other protective mechanisms in cancer patients and the human population. J Am Coll Nutr. 2001; 20(5 Suppl): 464S-475S.

12. Lamson DW, Brignall MS. Antioxidants and cancer, part 3: quercetin. Altern Med Rev. 2000; 5:304-329.

13. Christen Y. Oxidative stress in Alzheimer's disease. Am J Clin Nutr. 2000; 71:621-629 S.

14. Smith, MA, Perry G, Richery PL. Oxidative damage in Alzheimer's disease. Nature. 1996; 382: 120-121.

15. Age-Related Eye Disease Study Research Group. A randomized, placebo-controlled, clinical trail of high-dose supplementation with vitamins $\mathrm{C}$ and $\mathrm{E}$ and beta carotene for age related cataract and vision loss: AREDS report no.9. Arch Ophtalmol. 2001; 119:1439-1452.

16. Ehret A, Westendorp MO, Herr P, Debatin KM, Heeney JL, Frank R, et al. Resistance of chimpanzee $\mathrm{T}$ cells to human inmunodeficiency virus type 1 Tat-enhanced oxidative stress and apoptosis. J Virol. 1996; 70: 6502-6507.

17. Ortiz A, Vargas R, Muñoz G. Incidencia y mortalidad del cáncer en Costa Rica. Costa Rica: Kamelot Comunicación y Arte: 2005.

18. Siseles N. Información y formación para el manejo actual de la mujer en su climaterio. Buenos Aires: Ascune Hnos: 2005.

19. Stocker R, Keaney J. Role of oxidative modifications in atherosclerosis. Phsiol Rev. 2004; 84: 1381-1478.

20. Heinecke J. Oxidants and antioxidants in the pathogenesis of atherosclerosis: implications for the oxidized low density lipoprotein hypothesis. Atherosclerosis. 1998; 141: 1-15.

21. Salonen T, Yla-Herttulal S, Yamamoto R. Autoantibodies against oxidized LDL and progression of carotid atherosclerosis. Lancet. 1992; 339:883-887.

22. Gey KF, Puska P, Jordan P, Moser UK. Inverse correlation between plasma vitamin $\mathrm{E}$ and mortality from isquemia Herat disease in crosscultural epidemiology. ACJN. 1991; 53: 326-334 S.

23. Stampfer MJ., Hennekens CH, Manson J, Colditz G, Rosner B, Willet W. Vitamin E consumption and risk of coronary disease in women. New Engl J Med. 1993; 328: 1444-1449.

24. Stephens NG, Parsons A, Schofield PM, Kelly F, Cheeseman K, Mitchinson MJ, et al. Randomized controlled trail of vitamin E in patients with coronary disease: Cambridge Heart Antioxidant Study (CHAOS). Lancet. 1996; 347: 781-786.

25. Yusuf S. Vitamin E supplementation and cardiovascular events in high risk patients. New Engl J Med. 2000; 354: 1917-1918.

26. Lee IM, Cook NR, Gaziano J, Gordon D, Ridker P, Manson J, et al. Vitamin $\mathrm{E}$ in the primary prevention of cardiovascular disease and cancer: the Women's Health Study: a randomized controlled trail. JAMA. 2005; 294: 56-65.

27. Wassmann K, Wassmann S, Nickenig G. Progesterone antagonizes the vasoprotective effect of estrogen on antioxidant enzyme expression and function. Circ Res. 2005; 97: 1046-1054.

28. Strehlow K, Rotter S, Wassmann S, Adam O, Grohe C, Laufs K. et al. Modulation of antioxidant enzime expression and function by estrogen. Circ Res. 2003; 93: 170-177.

29. Thibodeau P, Kachadourain R, Lemay R, Bission M, Day B, Paquette B. In vitro pro- and antioxidant properties of estrogen. J Steroid Biochem Mol Biol. 2002; 81: 227-236.

30. Gorodeski G. Update on cardiovascular disease in post-menopausal women. Best Pract Res Clin Obstet Gynaecol. 2002; 16: 329-355.

31. Clarkson TB. The new conundrum: do estrogens have any cardiovascular benefits? Int J Fertil Womens Med. 2002; 47: 61-68.

32. Signorelli S, Neri S, Sciacchitano S, DiPino L, Costa M, Marchese G. Behaviour of some indicators of oxidative stress in postmenopausal and fertile women. Maturitas. 2006; 53: 77-82. 
33. Bednarek G, Tupikowski K, Bidzinska B, Bohdanowicz-Pawlak A, Antonowicz-Juchniewicz J, Kosouska B, et al. Serum lipid peroxides and total antioxidant status in postmenopausal women on hormone replacement therapy. Gynecol Endocinol. 2004; 19: 57-63.

34. Unfer T, Conterato M, da Silva J, Duarte M, Emanuelli T. Influence of hormonal replacement therapy on blood antioxidant enzymes in menopausal women. Clin Chem Acta. 2006; 369: 73-77.

35. Wen Y, Doyle M, Cooke T, Feely J. Effect of menopause on lowdensity lipoprotein oxidation: is oestrogen an important determinant? Maturitas. 2000; 34: 233-238.

36. Demirbag R, Yilmaz R, Erel O. The association of total antioxidant capacity with sex hormones. Scan Cardiovasc J. 2005; 36: 172-176.

37. Wells, G, Tugwell P, Shea B, Guyatt G, Peterson J, Zytaruk N. et al. Meta-analyses of therapies for postmenopausal osteoporosis. V. Metaanalysis of the efficacy of hormone replacement therapy in treating and preventing osteoporosis in postmenopausal women. Endocr Rev. 2002; 23:529-539.

38. Dick SE, DeWitt DE, Anawalt B. Postmenopausal hormone replacement therapy and mayor clinical outcomes: a focus on cardiovascular disease, osteoporosis, dementia and breast and endometrial neoplasia. Am J Manag Care. 2002; 8: 95- 104.

39. Manson JE, Hsia J, Johnson KC, Rossouw JE, Assaf AR, Lasser N, et al. Estrogen plus progestin and the risk of coronary heart disease. New Engl J Med. 2003; 349: 523.

40. Sugioka K, Shimosegawa Y, Nakano M. Estrogens as natural antioxidants of membrane phospholipid peroxidation. FEBS lett 1987; 210: 37-39.

41. Ayres S, Abplanalp W, Liu J, Ravi T. Mechanisms involved in the protective effect of estradiol -173 on lipid peroxidation and DNA damage. Am J Physiol. 1998; 274: E1002-E1008.

42. Subbiah MT, Kessel B, Agrawal M, Rajan R, Abplanalp W, Rymaszewski Z. Antioxidant potential of specific estrogens on lipid peroxidation. J Clin Endocrinol Metab. 1993; 77: 1095-1097.

43. Taniguchi S, Yanase T, Kobayashi K, Takayanagi R, Haji M, Umeda $\mathrm{F}$, et al. Catechol estrogens are more potent antioxidants than estrogens for the $\mathrm{Cu}(2+)$ catalyzed oxidation of low or high density lipoprotein: antioxidative effects of steroids on lipoproteins. Endocr J. 1994; 41:605-611.

44. Markides C, Roy D, Liehr G. Concentration dependence of prooxidant and antioxidant properties of catecholestrogens. Arch Biochem Biophys. 1998; 360: 105-112.

45. McManus J, McEneny J, Young IS, Thompson W. The effect of various oestrogens and progestogens on the susceptibility of low density lipoproteins to oxidation in vitro. Maturitas. 1996; 25: 125131.

46. Ayres SA, Tang M, Subbiah MT. Estradiol $17 \beta$ as an antioxidant: some distinct features when compared with common fat-soluble antioxidants. J Lab Clin Med. 1996; 128: 367-375.

47. Arteaga E, Rojas A, Villaseca P, Bianchi M, Arteaga A, Duran D. In vitro effect of estradiol, progesterone, testosterone and of combined estradiol/progestins on low density lipoprotein oxidation in postmenopausal women. Menopause. 1998; 5: 16-23.

48. Ke R, Todd D, Ahokas R. Effect of short-term hormone therapy on oxidative stress and endothelial function in African American and Caucassian postmenopausal women. Fertil Steril. 2003; 79: 11181122.
49. McManus J, McEneny, Thompson W, Young IS. The effect of hormone replacement therapy on the oxidation of low density lipoprotein in postmenopausal women. Atherosclerosis. 1997; 135: 73-81.

50. Inal M, Sunal E, Kanvak G, Zeytinoglu S. Effects of postmenopausal hormone replacement and alpha-tocopherol on the lipid profiles and antioxidant status. Clin Chem Acta. 1998; 268: 21-29.

51. Ozden S, Dildar K, Hakan Y, Guilzar K. The effects of hormone replacement therapy on lipid peroxidation and antioxidant status. Maturitas. 2001; 38: 165-170.

52. Naziroglu M, Simsek M, Simsek H, Aydilek N, Ozcan Z, Artilgan R. The effects of hormone replacement therapy combined with vitamins $\mathrm{C}$ and $\mathrm{E}$ on antioxidant levels and lipid profiles in postmenopausal women with Type 2 diabetes. Clin Chim Acta. 2004; 344: 63-71.

53. Wen Y, Doyle M, Norris L, Sinnott M, Cooke T, Harrison R, et al. Combined oestrogen-progesteron replacement therapy does not inhibit low-density lipoprotein oxidation in postmenopausal women. $\mathrm{Br} \mathrm{J}$ Clin Phamacol. 1999; 47: 315-321

54. Bureau I, Anderson R, Arnaud J, Raysifuier Y, Favier A, Roussel A. Trace mineral status in post menopausal women: impact of hormonal replacement therapy. J Trace Elem Med Biol. 2002; 16: 9-13.

55. Bureau I, Laporte F, Favier M, Faure H, Fields M, Favier A. et al. No antioxidant effect of combined HRT on LDL Oxidability and Oxidative stress biomarkers in treated post menopausal women. J Am Coll Nutr. 2002; 21: 333-338.

56. Halliwell B, Grootveld M, The measurement of free radical reactions in humans. FEBS lett 1987; 213 9-14.

57. Kaur I, Geetha T. Screening methods for antioxidants- a review. Mini Rev Med Chem. 2006; 6: 305-312.

58. Halliwell B, Clement MV, Long LH. Hydrogen peroxide in the human body. FEBS Lett 2000; 486: 10-13

59. Long LH, Evans PJ, Haliwell B. Hydrogen peroxide in human urine: implications for antioxidant defense and redox regulation. Biochem Biophys Res Commun. 1999; 262: 605-609.

60. Morrow JD, Hill KE, Burk RE. A series of prostaglandin $\mathrm{F}_{2}$-like compunds are produced in vivo in humans by non-cyclooxygenase, free radical-catalyed mechanism. Proc Natl Acad Sci USA. 1999; 87: 9383-9387.

61. Yagi K. Simple assay for the level of total lipid peroxides in serum or plasma. Methods Mol Biol. 1998; 108: 101-106.

62. Armstrong D, Browne R. The analysis of free radicals, lipid peroxides, antioxidant enzymes and compounds to oxidative stress as applied to the clinical chemistry laboratory. Adv Exp Med Biol. 1994; 366: 4358.

63. Stadtman ER, Oliver CN. Metal-catalyzed oxidation of proteins. Physiological consequences. J Biol Chem. 1991; 266: 2005-2008.

64. Beckman KB, Arnes B. Oxidative decay of DNA. J Biol Chem. 1997; 272: 19633-19636.

65. Kampa M, Nistikaki A. Tsaousis V. A new automated method for the determination of the total antioxidant capacity (TAC) of human plasma, based on the crocin bleachin assay. BMC Clinical Pathology. 2002; 2: 3-18. 